\title{
LAS ESTRATEGIAS DE SEGURIDAD DE LA UnIÓn EUROPEA $(2003,2016)$ : CONTEXTO POLÍTICO, CAMBIOS ESERCIALES Y EVOLUCIÓn DE LAS PRIORIDADES POLÍTICAS EUROPEAS RELATIVAS A ÁFRICA EN MATERIA DE SEGURIDAD
}

European Security Strategies $(2003,2016)$ :

Political Context, Essential Changes, and the Evolving European Policy Priorities Concerning African Security Issues 
In this article, we examine the most significant changes in the approach and formulation of the two European Union security strategies, presented in 2003 and 2016. This enables us to address fundamental issues related to the evolution of the European Union foreign and security policy since the beginning of the new millennium, in particular analysing the implications for the African continent. On the one hand, the analysis of the central elements of the two strategies allows us to observe a change of direction and a redefinition of the EU's foreign and security policy, from normative frameworks to newer approaches more focused on the objectives and interests of Europe. On the other hand, we can also observe the evolution of the European Union's priorities with regard to its approach to the African continent. In this respect, rather than addressing the different areas of mutual interest, the framework for relations between the EU and Africa is dependent on a EU's limited foreign policy approach, which is preponderantly focused on security issues in Africa.

\section{Introducción}

La característica principal de la política exterior europea ha sido y sigue siendo su naturaleza intergubernamental, donde rige la regla de la unanimidad, por lo que el aspecto clave de los procesos de toma de decisiones reside en la voluntad política de los Estados miembros (EE. MM.), según sus intereses nacionales a la hora de actuar o no actuar, y su concepción del uso de la fuerza y de la intervención externa. En función de esta premisa básica, el consenso de los Estados miembros condiciona la posición y la actuación de la Unión Europea (UE) en materia de política exterior, las iniciativas en relación con los temas globales más importantes y el establecimiento de mecanismos o instrumentos para avanzar (o no) en este sentido. En esta tesitura compleja, donde la unanimidad deviene en ocasiones, el principal factor limitante se sitúa en la construcción progresiva del rol internacional de la UE enmarcado en un enfoque integral u holístico de la política exterior y de seguridad.

La Estrategia Europea de Seguridad (EES), cuyo título versa: Una Europa segura en un mundo mejor, fue adoptada por la Unión Europea en diciembre de 2003 y representaba (hasta la formulación del documento de 2016), junto con el informe sobre su aplicación de diciembre de 2008, Ofrecer seguridad en un mundo en evolución, la dimensión exterior de la seguridad de Europa $^{1}$.

En un escenario global marcado por los acontecimientos del 11 de septiembre de 2001 y por la invasión de Irak de 2003, se intensificó el proceso de reflexión en torno a los ámbitos de la política exterior y de seguridad europea, con la adopción en diciembre de 2003 de la Estrategia Europea de Seguridad, donde se identifican determinadas amenazas prioritarias y objetivos

l No desarrollaremos en este trabajo la dimensión interior, plasmada en la Estrategia de Seguridad Interior de la Unión Europea: hacia un modelo europeo de seguridad, que fue adoptada por el Consejo de Justicia y Asuntos de Interior en su sesión de los días 25 y 26 de febrero de 2010 y aprobada por el Consejo Europeo en su reunión del 25 y 26 de marzo de 2010, y cuyos sendos informes sobre su aplicación se elaboraron en noviembre de 2011 y en abril de 2013. La Estrategia de Seguridad Interior de la UE: Hacia un modelo europeo de seguridad, remite de forma sintética a "los principales riesgos y amenazas para la seguridad a los que se enfrenta hoy Europa, como el terrorismo, la delincuencia organizada y grave, el tráfico de drogas, la ciberdelincuencia, la trata de seres humanos, la explotación sexual de menores y la pornografía infantil, la delincuencia económica y la corrupción, el tráfico de armas y la delincuencia transfronteriza, se adaptan muy rápidamente a la evolución científica y tecnológica, en su intento de aprovecharse ilegalmente y socavar los valores y la prosperidad de nuestras sociedades abiertas" (Consejo Europeo, 2010). Una Comunicación posterior de la Comisión al Parlamento Europeo y al Consejo, el 22 de noviembre de 2010, propuso cinco objetivos estratégicos con acciones específicas para poner en práctica la estrategia durante el periodo 2011 2014, "para superar los retos más urgentes y hacer que la UE sea más segura” (Comisión Europea, 2010). 
estratégicos, aunque el documento tiene una orientación demasiado general y no especifica detalladamente las líneas de acción concretas a seguir.

El también denominado "Documento Solana" pretendía ser una estrategia completa de seguridad y una referencia conceptual para la acción exterior de la Unión. Con la Estrategia, la Unión Europea aspiraba a dar un paso adelante introduciendo un "enfoque holístico", "integral" o "comprehensivo", que abarcara e integrase todas las dimensiones de la política exterior y superase los enfoques desarrollados en la pos Guerra Fría. La EES, teniendo en cuenta la incertidumbre creada después de los ataques del 11 de septiembre de 2001, tenía la intención de dar una respuesta convergente a la Estrategia de Seguridad Nacional de Estados Unidos (NSS), formulada en septiembre de $2002^{2}$. En este sentido, la EES significaba el intento de restablecimiento del vínculo transatlántico tras las tensiones creadas con el énfasis americano en el unilateralismo y en el uso de la fuerza, presentando prácticamente la misma definición de las amenazas que la NSS (Biscop, 2008, pp. 7-8; Tardy, 2009, p. 22).

No obstante, la EES hace hincapié en "un orden internacional basado en un multilateralismo eficaz", respetando el Derecho internacional, actuando según "el marco fundamental para las relaciones internacionales" que es la Carta de las Naciones Unidas y haciendo uso de medios tanto militares como civiles, incluyendo "la labor diplomática y las políticas de desarrollo, comercio y medio ambiente" (Consejo Europeo, 2003), para diferenciarse de la NSS y de su énfasis en el uso de medios coercitivos e instrumentos militares de forma unilateral, subordinando los enfoques multilaterales y el Derecho internacional a cuestiones de seguridad nacional y de integridad territorial (Biermann, 2009, p. 55).

La UE estaría promoviendo desde principios de siglo un enfoque holístico triangular, según el cual la seguridad, el desarrollo económico y los asuntos de gobernanza democrática son considerados "essential contributions to the generation of political stability in the EU's international environment” (Bagoyoko \& Gibert, 2009, p. 790). En términos reales, tanto la NSS como la EES supondrían una securitización de determinados aspectos de la política exterior, como el énfasis en la democracia, los derechos humanos o el comercio. Esta securitización representaría la instrumentalización de las dimensiones no militares de la política exterior para servir a intereses políticos o de seguridad, estando en el caso de la NSS en relación estrecha con la prioridad de la Guerra contra el Terror declarada por la Administración Bush tras los ataques del 11 de septiembre (Biscop, 2008, pp. 11-12).

La estrategia europea identifica cinco amenazas principales a las que debe enfrentarse la UE: el terrorismo, la proliferación de armas de destrucción masiva, los conflictos regionales, la descomposición del Estado y la delincuencia organizada. El documento recalca que "en esta época de globalización, las amenazas lejanas pueden ser tan inquietantes como las cercanas” y afirma la naturaleza dinámica de las nuevas amenazas en la pos Guerra Fría, y los cambios en la manera de afrontarlas: "Nuestro concepto tradicional de autodefensa, hasta el final de la Guerra Fría, se basaba en el peligro de invasión. Con las nuevas amenazas, la primera línea de defensa estará a menudo en el extranjero" (Consejo Europeo, 2003).

Al afirmar que la primera línea de defensa está en el extranjero, la Unión defiende la necesidad de una proyección de poder europeo hacia el exterior y de asumir responsabilidades mundiales en el ámbito de la paz y la seguridad, por lo que algunos autores consideran que la voluntad

2 La estrategia se conoce por el acrónimo NSS, derivado del título en inglés: US National Security Strategy.

\section{La estrategia europea recalca que "en esta época de globalización, las amenazas lejanas pueden ser tan inquietantes como las} cercanas" 
del documento es "a tacit calling for a more extrovert and active role of the Union in the world" (Haine, 2008, p. 21).

En esta misma línea, aunque de forma explícita y con un lenguaje más contundente, se sitúa el nuevo documento estratégico de junio de 2016, la estrategia global de la UE: Estrategia global para la política exterior y de seguridad de la Unión Europea: Una visión común, una actuación conjunta: una Europa más fuerte (EUGS)3. La nueva estrategia enuncia desde el prólogo la ambición de conseguir "una autonomía estratégica para la Unión Europea” y de contar "con la fuerza para contribuir a la paz y la seguridad en la región y en todo el mundo" (Servicio Europeo de Acción Exterior, 2016, pp. 3-4).

Por otro lado, el contenido y la propia estructura organizativa de la estrategia global, subdividida en epígrafes relativos a los intereses, los principios, las prioridades y la operatividad de la estrategia, reflejan la necesidad percibida en la UE de afirmarse como actor global con sus propios intereses y prioridades, además de defender una serie de principios y valores subyacentes a la propia construcción europea. Si bien los intereses se formulan de manera inextricablemente ligada a los valores fundamentales de la Unión, la retórica de integrar los intereses y los valores evidencia la orientación del proceso reflexivo llevado a cabo en la Unión en materia de política exterior y de seguridad en los últimos años, haciendo hincapié en los intereses europeos que inspiran el actual proceso.

La creciente aspiración de la Unión Europea de erigirse en un actor internacional creíble y con capacidad de respuesta, donde los valores estén respaldados por instrumentos y medidas concretas en seguridad y defensa, reflejan de esta manera el reconocimiento de las limitaciones del enfoque normativo de la Unión, como se explicita en la estrategia de 2016 presentada por la alta representante para Asuntos Exteriores y Política de Seguridad, Federica Mogherini:

En el mundo frágil en que vivimos, el poder simbólico no basta. Tenemos que potenciar nuestra credibilidad en materia de seguridad y defensa. Para hacer frente a las crisis externas, capacitar a nuestros socios y proteger Europa, los Estados miembros deben destinar recursos suficientes al gasto en defensa, hacer un uso lo más eficiente posible de los recursos y cumplir el compromiso colectivo de dedicar el $20 \%$ del presupuesto de defensa a investigación y tecnología y a la adquisición de material. (SEAE, 2016, p. 35)

En este artículo analizaremos la evolución de la política exterior y de seguridad de la Unión Europea desde el comienzo del nuevo milenio, estudiando fundamentalmente los cambios y continuidades más significativos entre las dos estrategias de seguridad de la UE, presentadas en 2003 y 2016. Nos centraremos en el análisis de la formulación de la política europea, con el objetivo de evidenciar si estamos ante una nueva orientación política, así como de elucidar los principales cambios conceptuales operados entre una estrategia de seguridad y otra. El análisis del impacto e implementación de las estrategias lo dejamos para un trabajo subsiguiente, por motivos de espacio. De esta manera, el estudio de los diferentes contextos de producción y de los elementos esenciales de los dos documentos estratégicos nos permitirá observar, por un lado, una serie de cambios conceptuales profundos que han contribuido a la redefinición de los enfoques y las políticas europeas de seguridad; y, por otro lado, la evolución de los intereses y las prioridades de la Unión en su aproximación al continente africano en materia de seguridad.

Los Estados miembros deben destinar recursos suficientes al gasto en defensa 


\section{La Estrategia Europea de Seguridad y la aproximación europea a Africa}

El nacimiento de la EES en 2003 se dio en uno de los momentos políticos más críticos para la Unión desde la guerra de los Balcanes, el momento de la guerra de Irak y la división de los entonces quince Estados miembros entre los que apoyaban la invasión más fervientemente (Reino Unido, España e Italia) y los que se oponían a ella (liderados por la posición de Francia y Alemania), demostrando las divergencias entre la UE y EE. UU., así como una profunda escisión europea y la inexistencia flagrante de una política exterior y de seguridad común ${ }^{4}$.

En 2003, la UE se encontraba además a un paso de llevar a cabo la ampliación más importante de sus Estados miembros, pasando de quince a veinticinco en 2004, divididos entre los que tenían una visión afín a la de EE. UU. en su consideración de Irak como Estado canalla, poseedor de armas de destrucción masiva, amenaza ante la cual era imperativo actuar por la fuerza; y los que rechazaban la invasión unilateral y preferían llevar el asunto de forma multilateral, bajo la autoridad de la ONU. La ampliación podía hacer bascular el equilibrio en el seno de la Unión hacia una posición más proatlantista, con lo cual, los Estados miembros vieron aconsejable llegar a un acuerdo en materia de seguridad antes de la expansión del proyecto de integración 5 .

La Estrategia Europea de Seguridad de 2003 comienza con una introducción en la que la Unión Europea manifiesta su ambición de convertirse en un actor global con responsabilidades "en el mantenimiento de la seguridad mundial y la construcción de un mundo mejor". Ya en el título de la estrategia se refleja esta voluntad, al situar la propia seguridad ("Una Europa segura") en relación o interdependencia con el contexto global ("en un mundo mejor"), en el que la UE desea intervenir. El documento presenta a continuación una estructura tripartita donde se expone el contexto de seguridad (desafíos mundiales y principales amenazas), los objetivos estratégicos y las implicaciones estratégicas para Europa, cerrando con una pequeña conclusión (Consejo Europeo, 2003).

El contenido de la EES, adoptada en 2003, ha sido comparado frecuentemente con el de la Estrategia de Seguridad Nacional de Estados Unidos publicada en septiembre de 2002, el cual constituye un documento de referencia para la elaboración de la EES al coincidir en algunos aspectos fundamentales como la identificación de las nuevas amenazas, a pesar de divergir en otros, como la forma de afrontarlas, desde un punto de vista unilateral vs. multilateral, dando protagonismo a medios coercitivos vs. una diversidad de instrumentos civiles y militares, y a la diferente importancia otorgada a las instituciones internacionales. Los elementos de conver-

4 La Comisión Europea, en contra de la invasión, manifestó su disgusto por la división de los Estados miembros en el seno del Consejo de Seguridad de Naciones Unidas (ONU). Por un lado, Francia, Alemania, Países Bajos, Luxemburgo, Suecia, Austria y Finlandia se mostraron en contra de la guerra, dada la ausencia de mandato bajo el Consejo de Seguridad de la ONU ni la autorización del uso de la fuerza. Por otro lado, Reino Unido, España, Portugal, Italia y Dinamarca se alinearon a favor de la intervención militar de la coalición en Irak (así como la mayoría de los países candidatos a la adhesión a la UE: Polonia, Hungría, Estonia, Letonia, Lituania, Eslovaquia, Bulgaria, República Checa y Rumanía). Grecia, Irlanda y Bélgica manifestaron también su oposición al conflicto, aunque Bélgica permitió la utilización de su espacio aéreo. Véase (Salmon, 2004, pp. 450-451).

$5 \mathrm{Al}$ margen del contexto político, algunos autores consideran que la adopción de la EES, diez años después de la institucionalización de la PESC, se hizo posible gracias a la existencia de un acervo de política exterior precedente en el que se apoya la EES, cuyo contenido se desarrolla en función de las áreas en las cuales los Estados miembros habían alcanzado un consenso previamente, dejando otros asuntos fuera o incluidos de forma deliberadamente vaga. En este sentido, la estrategia puede considerarse "a conceptual and procedural turning point" en el continuum del desarrollo de la PESC, así como un paso adelante "in the developing self-awareness and ambition of the EU as a player in the global arena”. Véase Bailes, 2005, p. 1.
La Estrategia

Europea de Seguridad de 2003 ha sido comparada frecuentemente con la Estrategia de Seguridad nacional de Estados Unidos de 2002 
gencia de la EES con la NSS responderían a la voluntad de restablecer el vínculo transatlántico después de la división de los Estados miembros con respecto a la cuestión iraquí, haciendo manifiesta la importancia de la relación entre Europa y EE. UU. para la seguridad mundial.

África se encuentra muy presente en el documento en el momento de identificación de las amenazas, aunque no de manera tan explícita como en el caso de la EUGS como veremos posteriormente. Las principales amenazas que identifica la estrategia europea son el terrorismo, la proliferación de armas de destrucción masiva, los conflictos regionales, la descomposición del Estado y la delincuencia organizada. La definición de las nuevas amenazas toma como referencia la nueva agenda de seguridad definida por EE. UU. en la NSS un año antes.

En el informe de revisión publicado cinco años después a la formulación de la estrategia, la UE añadió los retos de la ciberseguridad, la seguridad energética y el cambio climático. Asimismo, sitúa la proliferación de armas de destrucción masiva, el terrorismo y la delincuencia organizada en primera línea de interés, considerando necesaria una mejor conciliación de las dimensiones exterior e interior de la seguridad.

En la Estrategia Europea de Seguridad, la región de África Occidental se manifiesta como una zona de especial preocupación, en particular por la presencia de conflictos violentos sin resolver y por la amenaza a la seguridad que suponen los denominados Estados fallidos o frágiles: "Si se los descuida, los Estados en descomposición y la delincuencia organizada se extienden, como hemos podido ver en África Occidental”. De forma más general, África es caracterizada en el texto como un continente pobre, donde "en muchos casos, el fracaso económico está ligado a problemas políticos y a conflictos violentos” (Consejo Europeo, 2003).

Esta caracterización se reitera en el documento Estrategia de la UE para África de 2005, el cual supone "la respuesta de la Unión Europea al doble reto que consiste en volver a situar a África en la vía del desarrollo sostenible y en alcanzar los Objetivos de Desarrollo del Milenio (ODM) de aquí a 2015" (Comisión Europea, 2005, p. 44) ${ }^{6}$. Para lograr el desarrollo sostenible de África, la Unión Europea identifica las condiciones previas indispensables: la paz y la seguridad. Las crisis y los conflictos violentos presentes en África en las últimas décadas han terminado con millones de vidas y con varias décadas de desarrollo económico. Así pues, la UE explicita en la estrategia para África que "debería intensificar su ayuda en los ámbitos considerados como condiciones previas a la realización de los ODM; es decir, la paz, la seguridad y la buena gobernanza, necesarias para crear un entorno propicio al crecimiento económico, a los intercambios y a las interconexiones y a la cohesión social y medioambiental" (Comisión Europea, 2005, p. 4).

De la identificación de las amenazas prioritarias para la seguridad europea deriva la formulación de los tres objetivos estratégicos de la Unión, centrados en "hacer frente a las amenazas" enumeradas anteriormente, "crear seguridad en los países vecinos" para de esta forma garantizar la seguridad de la UE, y fomentar la existencia de "un orden internacional basado en un multilateralismo eficaz" para hacer frente a las amenazas a la seguridad internacional desde un sistema basado en el derecho y en el buen gobierno (Consejo Europeo, 2003).

Estos tres objetivos remiten de manera particular al continente africano, el cual es fuente de algunas de las principales amenazas para la seguridad de la Unión, que quiere rodearse de un círculo de países vecinos bien gobernados, hacia el este y hacia el sur, para evitar sufrir las re-
Las principales

amenazas identificadas son el terrorismo, las amas de destrucción masiva, los conflictos regionales, la descomposición del Estado y la delincuencia organizada 
percusiones de la desestabilización causada por los conflictos violentos y las consecuencias de los problemas de gobernanza.

En primer lugar, para hacer frente a las nuevas amenazas, la UE considera que, a diferencia de la situación de la época de la Guerra Fría, no basta con tener la capacidad militar adecuada: "Ninguna de las nuevas amenazas es meramente militar, ni puede atajarse únicamente con medios militares", sino que se necesita una combinación de instrumentos. En este sentido, la EES se distancia de la NSS, donde el uso de la fuerza militar es fundamental para afrontar los desafíos a la seguridad que acechan a los EE. UU. La UE, al haberse dotado tanto de capacidades militares como civiles para la gestión de crisis desde la institucionalización y operatividad de la Política Europea de Seguridad y Defensa (PESD), se muestra a sí misma como un actor idóneo para la gestión de crisis, cuya idiosincrasia sería precisamente poseer la combinación adecuada de los instrumentos cívico-militares para hacer frente a las nuevas amenazas: "La Unión Europea está especialmente preparada para responder a estas situaciones multidimensionales" (Consejo Europeo, 2003).

En segundo lugar, generar seguridad en los países vecinos se manifiesta como un objetivo estratégico fundamental en el contexto de la ampliación de la Unión prevista para 2004, poco después de la adopción de la EES: "La integración de los Estados adherentes aumentará nuestra seguridad, pero también acercará Europa a zonas conflictivas. Nuestra tarea es promover un conjunto de países bien gobernados al este de la Unión Europea y en las orillas del Mediterráneo [...]" (Consejo Europeo, 2003).

En este objetivo, la UE expresa de forma clara y contundente sus propios intereses de seguridad en relación con los países del este de Europa y de la ribera sur del Mediterráneo, a la hora de conseguir un vecindario bien gobernado, para evitar que el conflicto violento y la debilidad de los Estados alteren la estabilidad europea, al proliferar la delincuencia organizada, los problemas sociales o los fenómenos migratorios que podrían alterar el equilibrio interno de la Unión.

En tercer lugar, la UE inserta su acción en un sistema multilateral basado en el Derecho internacional, donde "el marco fundamental para las relaciones internacionales es la Carta de las Naciones Unidas". La UE, a diferencia del unilateralismo y excepcionalidad de las acciones americanas cuando se trata de la seguridad nacional, se define como un actor con vocación normativa que otorga una relevancia fundamental a las instituciones internacionales: "Queremos contar con organizaciones, regímenes y tratados internacionales eficaces para hacer frente a las amenazas a la paz y la seguridad internacionales, y debemos, por tanto, estar dispuestos a actuar cuando se vulneren sus normas" (Consejo Europeo, 2003).

Tras la formulación de los objetivos estratégicos de la UE, la EES termina con las implicaciones estratégicas para Europa, centrada en la voluntad de la UE de ser "más activos", "más capaces", "más coherentes", en colaboración "con nuestros socios". Si bien en el momento de formulación y adopción de la estrategia, la UE había recorrido un camino importante en el desarrollo de la política exterior y de seguridad común (PESC) y de la PESD; la operatividad de esta última, aunque declarada en Laeken, era todavía muy precaria, y no se habían alcanzado los objetivos definidos en los objetivos principales (Headline Goals) sobre las capacidades militares y civiles. Es en este contexto intraeuropeo en el que la UE declara su ambición, todavía irrealizada, de devenir un actor de seguridad.

Para ello se explicita la necesidad de ser más activos en el uso de la panoplia de instrumentos militares y civiles, además de en las actividades tradicionales en el ámbito del comercio, el de- 
sarrollo y la diplomacia, y en el apoyo a las acciones de la ONU, con el objetivo de adquirir un mayor peso político y presencia internacional que contribuya a su identidad como actor: "Una Unión Europea que asuma una mayor responsabilidad y que desempeñe un papel más activo tendrá mayor peso político”. En estrecha relación, la UE manifiesta su voluntad de ser más capaz, desarrollando los recursos militares y sobre todo civiles para hacer frente a las nuevas amenazas, aunque no se especifica de qué forma ni qué capacidades serían necesarias para afrontar cada amenaza o cómo está previsto combinar los recursos, simplemente se menciona de forma vaga e inespecífica. Por otro lado, la UE manifiesta su voluntad de ser más coherente, asociando y relacionando las actividades de los diferentes pilares y las acciones exteriores de los Estados miembros, aunque nuevamente no se especifica ninguna acción concreta o específica.

La estrategia se cierra con una breve apología del multilateralismo, separándose de la visión unilateralista americana: "Pocos son los problemas, si es que hay alguno, a los que podamos hacer frente en solitario. Las amenazas a las que hemos aludido son amenazas comunes, que compartimos con nuestros socios más cercanos. La cooperación internacional es un imperativo”, y expone la necesidad de estrechar relaciones con los socios internacionales, generando asociaciones estratégicas "con todos los países que comparten nuestros objetivos y nuestros valores y estén dispuestos a apoyarlos”, realzando el discurso sobre la vocación normativa de la Unión para conseguir "un mundo más justo, más seguro y más unido” (Consejo Europeo, 2003).

\section{La estrategia global para la política exterior y de seguridad de la Unión Europea: contexto histórico y político}

En 2003, la UE estaba inmersa en un proceso global de reflexión, enmarcado en la Convención Europea de 2002-2003, y la Conferencia Intergubernamental subsiguiente, cuyo objetivo era redactar una Constitución Europea. En este contexto institucional, y con ánimo de reparar la desunión creada por la crisis iraquí, fomentando el consenso y la cohesión entre los Estados miembros, la UE consideró necesario articular las bases conceptuales de la PESC y de la PESD, cuyo desarrollo se había minimizado hasta el momento.

Los distintos acontecimientos políticos internos y globales mencionados más arriba llevaron a los Estados miembros a encomendar al alto representante, durante una reunión informal de ministros de asuntos exteriores en Kastellorizo en mayo de 2003, la formulación de un "concepto estratégico europeo". Solana y su equipo presentaron una primera versión ante el Consejo en junio de 2003, y el documento estratégico final se adoptó en el Consejo Europeo el 12 de diciembre de 2003 en Bruselas. El documento se sometió a discusión interna y externa. Tres conferencias de investigación celebradas en los meses de septiembre y octubre bajo la égida del Instituto de Estudios de Seguridad de la Unión Europea (EUISS) proveyeron al documento con la visión y feedback de expertos y académicos internacionales ${ }^{7}$. Posteriormente, las discusiones prosiguieron a nivel de los Estados miembros y de la comisión, realizando las modificaciones necesarias, hasta la adopción en diciembre del documento de forma consensuada (Bailes, 2005, pp. 10-11).

\section{La estrategia se cierra con una breve apología del multilateralismo y la cooperación intemacional}


Esta modalidad de discusión y revisión del texto, tomando en consideración tanto a los Estados miembros como a la Comisión, y a la comunidad científica y académica de los estudios de seguridad, refleja un nuevo patrón procedimental más colectivo, con ánimo de inclusión de los diferentes actores implicados y con el objetivo de generar aceptación y visibilidad en aras de lograr legitimidad, así como un amplio apoyo político para minimizar los trastornos del disenso. En palabras de Bailes:

The EU community was starting to realize how important it could be (for European credibility and impact) not just to produce the right message but to be able to get it across, in the right words and at the right time, with the right intellectual allies. (Bailes, 2005, p. 12)

Este procedimiento de consulta y discusión, tanto interna como externa, se ha llevado a cabo asimismo con la estrategia global de la Unión, que ha generado un amplio debate entre los expertos y académicos, además de discutirse en el seno de las instituciones europeas. Diez años después de la formulación de la EES, en el Consejo Europeo de diciembre de 2013, se encomendó a la alta representante y vicepresidenta de la comisión, Federica Mogherini, la revisión estratégica del documento, el análisis de los cambios y transformaciones operados en el escenario mundial y la evaluación sobre tales cambios y sobre los desafíos para la Unión Europea como actor en el ámbito de la política exterior y de seguridad.

\section{En la estrategia} se reorientan las prioridades hacia problemas de índole económica y doméstica

A este proceso de reflexión y revisión, culminado en 2015, siguió la fase formal de elaboración de una nueva estrategia que reflejara la posición de la UE ante los cambios internacionales y determinara su ambición como actor global, estrategia presentada por Mogherini a los líderes europeos el 28 de junio de 2016.

El proceso de reflexión y elaboración de la EUGS ha contado con el interés e implicación de múltiples especialistas en la materia, los cuales han publicado informes y análisis sobre la evolución de la política exterior y de seguridad europea. De igual manera, se han celebrado de manera periódica conferencias de expertos en el seno del Instituto de Estudios de Seguridad de la Unión Europea, quienes proveen una importante realimentación en cuanto a los procesos de desarrollo, revisión y evaluación de las políticas europeas de seguridad y defensa.

No obstante, el interés preeminente que se le dio a la formulación de los conceptos estratégicos entre 2003 y 2008, fecha de actualización de la EES, no prosiguió en los años posteriores. Se abandonó de esta manera el foco en la renovación de la estrategia durante el periodo 20092014, abordando otros temas de actualidad en una Unión inmersa en la crisis económica de carácter mundial ${ }^{8}$.

A partir de 2008, el contexto de la crisis financiera y económica a escala internacional ha supuesto sin duda uno de los factores más importantes que han marcado un cambio en el eje de interés de la UE, en el seno de la cual se había llevado a cabo, desde el año 2000, un proceso de construcción y reformulación de la política exterior y de seguridad. Sin abandonar el interés en los temas exteriores y de seguridad, se reorientaron las prioridades hacia problemas de índole económica y hacia preocupaciones domésticas.

8 Un análisis más detallado de los temas y contenidos de las diferentes conferencias anuales celebradas en el EUISS (European Union Institute for Security Studies) puede verse en La nueva Estrategia de Seguridad Europea (De Carlos Izquierdo, 2016). 
Ante este contexto poco favorable para la reflexión y reformulación de los conceptos estratégicos europeos y para la definición de las prioridades exteriores de la Unión, hubo que esperar a finales de 2013 para ver el resurgir de la relevancia de los asuntos de conceptualización de la seguridad. Es en 2013 cuando el Consejo Europeo encomendó a Mogherini la revisión de la estrategia de la Unión, y también es el momento de publicación de un documento fundamental sobre el denominado concepto integral: El enfoque integral adoptado por la UE en relación con los conflictos y las crisis exteriores (Comisión Europea y Alta Representante de la UE, 2013).

El "enfoque integral" de la UE o comprehensive approach es un concepto que remite al "enfoque multidimensional" de Naciones Unidas. Ambas organizaciones han desarrollado progresivamente en las últimas décadas un enfoque holístico como guía de su actividad en el ámbito de la seguridad, de forma particular en lo referente a la gestión de crisis y la resolución de conflictos.

A pesar de esta reactivación del proceso, un evento fundamental e inesperado vino a empañar la culminación del proceso de reflexión conducido entre 2013 y 2015. El momento de presentación de la nueva estrategia global de la UE pasó, si no desapercibido, sí desatendido por el terremoto político que supuso el denominado brexit (abreviatura de British exit), o bien, la celebración de un referéndum sobre la permanencia de Reino Unido en la UE, evento que ha tambaleado de forma duradera los cimientos de la UE, generando una crisis interna que ha obligado a la Unión a repensar el mismo proyecto de integración europea, los parámetros políticos que la guían e incluso su mismo sentido y supervivencia.

A raíz del resultado de la votación del 23 de junio de 2016 que acabó en la salida de Reino Unido de la Unión, la presentación formal de la nueva EUGS el 28 de junio de 2016 quedó en un segundo plano, ensombreciendo el trabajo de reflexión de los últimos años, que pretendía reavivar el interés europeo en los temas referentes a la seguridad y la defensa.

Tendremos que esperar un tiempo prudencial para ver si el contexto desfavorable de elaboración y presentación de la nueva estrategia de la UE supone un hándicap para la implementación de la misma, o si, por el contrario, las consecuencias que comporta el brexit determinan para la Unión una reacción en el sentido de lograr mayor cohesión entre los Estados miembros a nivel político, y una voluntad de actuar a nivel internacional de manera conjunta y consistente.

Estas preocupaciones internas e institucionales, junto con la importancia de los eventos internacionales y la transformación del orden mundial, suponen desafíos de primer nivel que la Unión debe afrontar, renovando de forma periódica el concepto estratégico y redefiniendo las prioridades de acción para no correr el riesgo de elaborar formulaciones tardías que no reflejen las reacciones a los rápidos cambios ocurridos en el entorno estratégico global, pudiendo resultar por tanto de naturaleza obsoleta.

\section{4. nuevos planteamientos y cambios en las prioridades para el continente africano en la estrategia global de la Unión Europea}

La Estrategia Europea de Seguridad de 2003 especificaba de forma directa el conjunto de amenazas y desafíos a la seguridad que la UE consideraba prioritarios en la nueva tesitura internacional generada tras el final de la Guerra Fría. A nivel global, la UE consideraba que los cambios

\section{La UE ha} desarrollado un enfoque holístico como guía de su actividad en el ámbito de la seguridad 
en el contexto de seguridad de la pos Guerra Fría y de la mundialización habían traído consigo una mayor interdependencia entre los aspectos internos y externos de la seguridad, así como la vinculación entre la seguridad y el desarrollo. Por ende, la UE, en su afán de constituirse en un actor de seguridad, manifestaba la necesidad de actuar en coordinación con otros actores internacionales, interviniendo en distintos sectores y áreas políticas:

La seguridad es una condición para el desarrollo. El conflicto no solo destruye las infraestructuras, incluidas las sociales, sino que también fomenta la delincuencia, disuade a los inversores e imposibilita la actividad económica normal. Varios países y regiones han quedado atrapados en un ciclo de conflicto, inseguridad y pobreza. (Consejo Europeo, 2003, p. 2)

Existen importantes cambios en el documento de la estrategia global de la UE de 2016 respecto a la estrategia de 2003, los cuales muestran la evolución de la reflexión europea en torno a las cuestiones de seguridad y defensa. En este epígrafe intentaremos abordar por un lado las principales novedades en los conceptos y en los planteamientos estratégicos, que hemos subdividido en tres aspectos cruciales, para definir preliminarmente las grandes líneas de reorientación de la política exterior y de seguridad de la UE; por otro lado, estudiaremos cuál es el lugar de África en la nueva estrategia y su prominencia en las áreas prioritarias de la acción exterior de la UE.

Un primer aspecto que llama la atención al comparar los dos documentos estratégicos es el énfasis otorgado en la EUGS a los propios intereses de la Unión. En contraposición con la insistencia en los valores y principios de la EES, en el documento actual, la UE deja claro desde el comienzo que la EUGS tiene como objetivo promover los intereses de los ciudadanos de la Unión. Aunque evidentemente no se abandonan los principios y los valores tradicionales de la Unión", estos quedan impregnados de intereses europeos: "Nuestros intereses y nuestros valores van de la mano. Redunda en nuestro interés promover nuestros valores en el mundo. Al mismo tiempo, nuestros valores fundamentales están integrados en nuestros intereses" (SEAE, 2016, p. 10).

De esta forma, se adopta públicamente una concepción más realista de las relaciones internacionales, donde la política exterior se formula en términos realistas derivados de la evaluación del entorno estratégico, de acuerdo con un "pragmatismo basado en principios", nuevo concepto introducido en la EUGS, que sin una definición específica parece responder a esta interacción entre realismo e idealismo en el discurso de la política exterior y de seguridad europea.

No obstante, la relevancia otorgada a los propios intereses no puede ser considerada una novedad stricto sensu, debido a que, en la práctica, la política exterior de la Unión ha promovido siempre sus propios intereses al desempeñar su papel como actor internacional en el mundo. El aspecto que sí supone una novedad interesante es la afirmación pública y contundente de los intereses, en detrimento del habitual discurso normativo sobre la fundamentación europea en principios y valores. El documento adopta un lenguaje más directo y más realista, en consonancia con los cambios operados en el entorno geoestratégico actual que han afectado directamente a Europa hasta cuestionar el propio proyecto de integración europea: "Vivimos una época de crisis existencial, dentro y fuera de la Unión Europea. Nuestra Unión está ame-
Aunque no se abandonan los principios y los valores tradicionales de la Unión, quedan impregnados de intereses europeos

9 Los valores, integrados en los intereses vitales, son la paz, la seguridad, la prosperidad, la democracia y un orden mundial basado en normas, mientras que los principios rectores de la acción exterior de la UE son la unidad, la interacción, la responsabilidad y la asociación. Véase (SEAE, 2016, p. 12). 
nazada. Nuestro proyecto europeo, que ha traído un periodo de paz, prosperidad y democracia sin precedentes, está siendo cuestionado” (SEAE, 2016, p. 10). Consecuencia directa de este nuevo enfoque más realista es la prioridad otorgada a la seguridad de la UE, segundo aspecto que llama la atención del nuevo documento estratégico. Si en el documento de 2003 la valoración de las amenazas se hacía en función de cómo afectaban a la gobernanza mundial, en 2016 la consideración de las amenazas se centra en cómo pueden afectar a la UE.

En relación con este punto se introduce un elemento que ha ocupado un lugar central en las discusiones europeas en materia de política exterior y de seguridad, el hecho de adquirir una capacidad propia en materia de defensa y contribuir a la seguridad colectiva de Europa. Se manifiesta de esta manera la necesidad de "asumir una mayor responsabilidad en nuestra seguridad" y de tener "un nivel adecuado de ambición y autonomía estratégica”. La introducción de este concepto de "autonomía estratégica” es fundamental, refleja la evolución de los debates en el seno de la Unión y la voluntad de avanzar en materia de defensa, para "garantizar la seguridad dentro y fuera de sus fronteras" (SEAE, 2016, p. 14).

Las principales amenazas que la Unión identifica para su seguridad son el terrorismo, las amenazas híbridas, la volatilidad económica, el cambio climático y la inseguridad energética. El documento ahonda en la amenaza que supone el terrorismo y la violencia en el norte de África y en Oriente Próximo, y en su expansión hacia la propia Europa, donde se han perpetrado importantes atentados terroristas.

Al hablar de terrorismo como una de las principales amenazas a la seguridad de la UE, se hace mención expresa al norte del continente africano y a la necesidad de luchar contra el extremismo violento. No es la única mención al continente en la EUGS, sino que podemos observar que África está mucho más presente explícitamente en la actual estrategia de lo que encontramos en la estrategia precedente.

El continente africano está presente, de hecho, de diferentes maneras, en cada una de las cinco áreas definidas como prioritarias para la acción exterior de la Unión. En primer lugar, como fuente de amenazas que pueden afectar a la seguridad de Europa, particularmente en forma de extremismo violento, radicalización y redes terroristas. En segundo lugar, como foco primordial donde fortalecer la resiliencia estatal y de la sociedad por parte de la UE. La resiliencia es un concepto en boga en las ciencias sociales que la UE no ha dudado en adoptar en su discurso y que se plantea aplicar en el continente africano, en la región vecina de África del Norte, hasta África Central, para favorecer la transformación de la gobernanza hacia regímenes democráticos, intensificar las relaciones con la Unión y evitar la fragilidad que conduce a la movilidad y la migración ilegal que afectan a las fronteras de Europa.

En tercer lugar, en lo que respecta al enfoque integral para atajar los conflictos y las crisis, los conflictos armados africanos, en particular en la región del Sahel y del Cuerno de África, pueden amenazar los intereses europeos, por lo que la UE se compromete a aplicar un enfoque pluridimensional "mediante la utilización de todos los instrumentos y políticas disponibles orientados a la prevención, la gestión y la resolución de conflictos"; un enfoque de múltiples fases, "actuando en todas las fases del ciclo del conflicto"; un planteamiento de múltiples niveles, "actuando a escala local, nacional, regional y mundial", y un enfoque multilateral, "que comprometa a todos los actores que estén presentes en un conflicto y resulten necesarios para su resolución” (SEAE, 2016, pp. 22-23). 
En cuarto lugar, en cuanto al apoyo y la cooperación con las organizaciones regionales, consideradas fundamentales por la UE para mejorar la gobernanza regional y mejorar la gestión de las crisis ("es una de las lógicas fundamentales subyacentes a la paz y el desarrollo propios de la UE en el siglo XXI”), África ocupa un lugar muy destacado, tanto en el ámbito de la seguridad: "a la luz de las crecientes interconexiones entre África del Norte y el África Subsahariana, así como entre el Cuerno de África y Oriente Próximo, la UE apoyará la cooperación a través de estas subregiones. Ello incluye el fomento de las relaciones triangulares a través del Mar Rojo entre Europa, el Cuerno de África y el Golfo, a fin de hacer frente a los desafíos para la seguridad y aprovechar las oportunidades económicas. Ello supone abordar de modo sistemático la dinámica transfronteriza en el norte y el oeste de África, el Sahel y la región del Lago Chad a través de vínculos más estrechos con la Unión Africana, la Comunidad Económica de los Estados del África Occidental (CEDEAO) y el G5 del Sahel”; así como en la esfera del desarrollo:

Invertiremos en paz y desarrollo para África como inversión en favor de nuestra propia seguridad y prosperidad. Intensificaremos la cooperación con la Unión Africana, y también la CEDEAO, la Autoridad Intergubernamental para el Desarrollo del África Oriental y la Comunidad del África Oriental, entre otras organizaciones, así como la ayuda en su favor. Debemos redoblar nuestros esfuerzos para estimular el crecimiento y el empleo en África. (SEAE, 2016, p. 28)

Por último, en quinto lugar: "La UE está comprometida con un orden mundial basado en el Derecho internacional, incluidos los principios de la Carta de las Naciones Unidas, que asegure la paz, los derechos humanos, el desarrollo sostenible y un acceso constante al patrimonio natural de la humanidad”, y en este sentido África es también parte del escenario global donde la UE pretende actuar y aspira a transformar el sistema internacional y los patrones de la gobernanza global en el siglo XXI.

En estas cinco áreas prioritarias, África se muestra como un continente fundamental para la Unión y no como un desafío secundario a la seguridad, aunque la estrategia no especifica cómo se hará frente a los desafíos enumerados ni las modalidades concretas de implantación, por lo que la valoración de las ambiciones expresadas en el papel resulta imposible y será necesario observar el contenido y el establecimiento de los planes de acción que se pongan en marcha al respecto, para evaluar la coherencia y la consistencia con los planteamientos enumerados.

Un tercer aspecto de la EUGS es la atención al enfoque holístico o integral en relación con los conflictos y las crisis, y la voluntad europea de comprometerse en la construcción de la paz, de manera particular en las regiones vecinas del este y el sur de la Unión. La plasmación del enfoque integrado es la continuación lógica de la vinculación entre la seguridad y el desarrollo afirmada ya en la EES. La EUGS adopta el enfoque integrado, definido en 2013, y enfatiza en llevar a cabo acciones de prevención, gestión y resolución de conflictos, a diferencia del hincapié de la EES en la prevención de conflictos:

Es esencial aplicar un enfoque pluridimensional mediante la utilización de todos los instrumentos y políticas disponibles orientados a la prevención, la gestión y la resolución de conflictos. Pero el alcance del "enfoque global” se extenderá aún más. No existen soluciones rápidas para ninguno de estos conflictos. La experiencia adquirida en Somalia, Malí, Afganistán y otros países pone de relieve su naturaleza prolongada. La UE tratará de plasmar por consiguiente un enfoque de múltiples fases, actuando en todas las fases del ciclo del conflicto. Invertiremos en la prevención, la resolución y la estabilización, y 
evitaremos el cese prematuro de nuestro compromiso cuando una nueva crisis estalle en otro lugar. (SEAE, 2016, p. 22)

Los tres aspectos fundamentales de la Estrategia están interrelacionados: los intereses de la UE, la seguridad de la UE y la voluntad de la UE de actuar en el mantenimiento de la paz. La adquisición de nuevas capacidades en el ámbito de la defensa puede, en este sentido, permitir a la Unión actuar en todas las fases de los conflictos y en la gestión de crisis, colaborando así con las acciones internacionales de construcción de la paz, mientras que la mejora en la situación de seguridad revierte en la seguridad interior de la Unión y en proteger sus intereses y los de sus ciudadanos.

\section{Conclusión}

En este artículo hemos abordado la evolución de la política exterior y de seguridad de la Unión Europea desde el comienzo del nuevo milenio, centrando el análisis en el estudio de los cambios más significativos existentes en el planteamiento y formulación de las dos estrategias de seguridad de la UE, presentadas en 2003 y 2016.

De acuerdo con nuestro análisis, podemos afirmar que existen importantes cambios conceptuales entre los dos documentos estratégicos analizados, lo cual evidenciaría un cambio de rumbo en la política exterior y de seguridad europea, al menos en lo que respecta a su formulación y definición en el nuevo milenio. El examen de los elementos centrales de las dos estrategias nos permite observar una redefinición progresiva de la política exterior y de seguridad de la UE, desde presupuestos normativos hasta aproximaciones más centradas en los objetivos e intereses propios de Europa.

En este sentido, la estrategia global de la Unión Europea de 2016 deja en un segundo plano el enfoque normativo de la Unión y su trasfondo idealista, para manifestar claramente la preponderancia de sus intereses, comenzando por el ámbito prioritario de garantizar la seguridad de la UE. Esta aproximación de corte más realista refleja en el papel la visión de la Unión y la reorientación de la política exterior y de seguridad en términos más contundentes que superan el marco del poder blando, aunque no se especifica la manera concreta de implantación y los indicadores de seguimiento.

A nivel conceptual, existen diferencias significativas entre las dos estrategias. Al describir las amenazas y retos a la seguridad, el análisis de la EES hace hincapié en la interrelación entre los aspectos económicos, sociales y políticos como fuente de los conflictos violentos y la inseguridad, según un enfoque posmoderno o poswestfaliano de la gobernanza mundial; mientras la EUGS, marcada por los ataques terroristas perpetrados en suelo europeo, enfatiza en su discurso la existencia de amenazas que pueden afectar a los intereses de la UE en una óptica más tradicional, realista y westfaliana. La EES prefiere extenderse en la definición de las principales amenazas y sus consecuencias para la gobernanza mundial, mientras que la EUGS focaliza el análisis en los riesgos que pueden acarrear los conflictos armados, el terrorismo o la inseguridad energética para la propia seguridad de la UE.

A este respecto, la UE insiste en 2003 en la importancia de la prevención de conflictos, estando el documento imbuido de referencias al multilateralismo y a la acción internacional enmarcada en torno a las instituciones internacionales responsables del mantenimiento de la paz y seguridad internacionales, mientras que en 2016 se habla del enfoque integrado y de llevar a cabo

\section{La EUGS enfatiza la existencia de amenazas que pueden afectar a los intereses de la UE en una óptica más tradicional, realista y mestfaliana}


acciones en todas las etapas del ciclo del conflicto, no solo en la fase de prevención y de adquirir capacidades para poder desarrollar acciones militares, asumiendo un mayor compromiso y responsabilidad a la hora de responder a las crisis y conflictos violentos internacionales.

Asimismo, la Estrategia Europea de Seguridad de 2003 ahonda en conseguir el objetivo de crear un orden internacional basado en un multilateralismo eficaz. Sin embargo, el compromiso de la Unión con el desarrollo de una sociedad internacional fuerte y de un orden internacional basado en el derecho, donde las instituciones internacionales funcionen adecuadamente, se matiza en la estrategia global de la UE de 2016. La vocación normativa de la UE, manifestada en la implementación de sus principios y valores, se enuncia en relación con los intereses europeos en términos de estabilidad y de seguridad, ejes prioritarios de la acción exterior europea. Los intereses europeos son, por tanto, los factores clave a la hora de contribuir al desarrollo de la gobernanza global y de las organizaciones regionales e internacionales.

Por otra parte, la estrategia global de la UE de 2016 supone un salto cualitativo importante respecto de su predecesora en lo que se espera de un documento estratégico, comenzando por la utilización de un lenguaje más preciso en materia de seguridad y defensa, seguido de la importancia otorgada a la autonomía estratégica y la adquisición de capacidades militares, y terminando por la voluntad de implicación en los procesos de resolución de conflictos y gestión de crisis internacionales.

En cuanto al segundo componente fundamental de nuestro análisis, podemos también observar la evolución, en el periodo de tiempo examinado, de los intereses y las prioridades de la Unión Europea en lo que respecta a los planteamientos y al enfoque integral sobre los asuntos de seguridad en el continente africano (de forma coherente y convergente con el cambio de rumbo en la política exterior y de seguridad de la UE enunciado más arriba).

En el ámbito geográfico de África, la visión de la UE en cuanto a la percepción sobre el continente africano y sobre los desafíos a la seguridad que de él emanan, constituye una visión bastante limitada e imbuida de la vinculación entre las dimensiones relacionadas con la seguridad y el desarrollo (aproximación que encuentra su culminación en el desarrollo del enfoque integrado hacia los conflictos), en el seno de un planteamiento de carácter liberal, que privilegia la búsqueda de la seguridad y justifica de esta manera el desarrollo de un tipo de intervencionismo en pos de un proceso de (re)construcción de las estructuras del Estado que garanticen la estabilidad y eviten la diseminación de las amenazas.

África se percibe como fuente de amenazas que pueden afectar a la seguridad de la UE. En este sentido, las acciones emprendidas por la UE en el continente tienen como objetivo directo evitar que tales desafíos repercutan en los intereses de la UE y comprometan la seguridad de la Unión. La identificación de las amenazas, así como las acciones y las políticas propuestas, siguen un diagrama circular donde las iniciativas se originan y revierten en la propia seguridad de la Unión.

Ante el creciente fenómeno de radicalización y terrorismo, la UE afirma su voluntad de invertir en la resiliencia estatal y de la sociedad de los países vecinos del sur del Mediterráneo, hasta África Central. La resiliencia o "capacidad de los Estados y las sociedades para reformarse, aguantando así los desastres, y para recuperarse de las crisis internas y externas” (SEAE, 2016, p. 18) es fundamental en el discurso europeo para garantizar la existencia de Estados seguros, prósperos y democráticos. De esta manera, la resiliencia estatal y de la sociedad podría evitar 
la fragilidad de las instituciones y facilitar el buen gobierno, con la consecuencia directa para Europa de disminuir los flujos migratorios y los atentados que afectan a la seguridad interior de la UE.

Sin embargo, el discurso relativo a África y la preocupación acerca del terrorismo, las migraciones, los conflictos violentos, el subdesarrollo y la debilidad de las estructuras estatales, no se ve acompañado de la formulación de una línea de acción coherente y específica, generándose una brecha entre los objetivos ambiciosos en el papel en términos de seguridad y la limitada atención que se puede o quiere prestar al continente.

De igual forma, los conflictos violentos que se dan en el continente africano tienen consecuencias a nivel nacional, regional y global, que la UE pretende atajar mediante políticas de prevención y resolución de conflictos. No obstante, el protagonismo cada vez mayor otorgado al apoyo y fortalecimiento de las organizaciones regionales africanas implica en términos prácticos la ambición de delegar las responsabilidades en materia de seguridad a tales organizaciones regionales, para que puedan llevar adelante sus cometidos de manera autónoma, reduciendo la participación directa y la implicación europea (según el lema "soluciones africanas para los problemas africanos").

Además, las prioridades de la Unión Europea en África se enuncian en la EUGS únicamente en relación con los problemas de seguridad, cuando en otros documentos, como en la Estrategia Conjunta UE-África, adoptada en 2007, se abordan las relaciones de manera integral en función de áreas prioritarias de cooperación que abarcan los asuntos de paz y seguridad, el desarrollo sostenible, la gobernanza democrática y los derechos humanos, la integración regional y el comercio, el cambio climático y la energía, la movilidad y las migraciones o la cooperación científica.

En 2017 se celebró la V Cumbre UE-África y la ambición de los líderes africanos se situó en dialogar sobre todas las áreas de cooperación, y en adoptar un enfoque a largo plazo, y no únicamente en concentrar el interés en el área de la seguridad o en las amenazas que Europa percibe como inherentes al continente africano. Por otra parte, se acerca también el final del periodo de vigencia del Acuerdo de Cotonú, firmado en 2000, con una validez de veinte años, por lo que este marco tradicional de relaciones de la UE con los denominados países ACP (África, Caribe y el Pacífico) ha de remodelarse.

Las limitaciones en el enfoque de política exterior de la UE hacia el continente africano, centrado de manera preponderante en los asuntos de seguridad, puede tener a medio y largo plazo consecuencias negativas para los intereses políticos y económicos de la Unión, así como para su seguridad. De hecho, las medidas militares por sí mismas no son suficientes en la lucha contra el terrorismo y el radicalismo, ya que no pueden abordar las causas estructurales del extremismo en África. Las relaciones entre la UE y África deben reformularse para abordar los diferentes ámbitos de interés mutuo.

En este momento de cambio y reformulación de las políticas africanas de la Unión, se debe hacer un esfuerzo para que la definición de las relaciones entre la UE y África no se centre única o principalmente en los enfoques y las prioridades europeas, sino que desarrolle un diálogo entre socios iguales, evitando la reproducción de modelos de relación asimétricos ya conocidos.

\section{Las medidas militares por sí mismas no son suficientes en la lucha contra el terrorismo y el radicalismo}




\section{Referencias}

Bagoyoko, N., \& Gibert, M. V. (2009). The Linkage between Security, Governance and Development: The European Union in Africa. The Journal of Development Studies, 45(5). DOI: https://doi.org/10.1080/00220380802582312

Bailes, A. J. K. (2005). The European Security Strategy: An Evolutionary History. SIPRI Policy Paper, (10).

Biermann, R. (2009). NATO's Institutional Decline in Post-Cold War Security Governance. En C. Wagnsson, J. A. Sperling, \& J. Hallenberg (Eds.), European Security Governance: The European Union in a Westphatian World. London, United Kingdom: Routledge.

Biscop, S. (2008). The European Security Strategy in Context: A Comprehensive Trend. En S. Biscop \& J. J. Andersson (Eds.), The EU and the European Security Strategy: Forging a Global Europe. London: Routledge.

Comisión Europea. (2010, noviembre, 22). Comunicación de la Comisión al Parlamento Europeo y al Consejo: La Estrategia de Seguridad Interior de la UE en acción: Cinco medidas para una Europa más segura. Bruselas.

Comisión Europea. (2005). Estrategia de la UE para África: Hacia un pacto euroafricano para acelerar el desarrollo en África. Bruselas.

Comisión Europea y Alta Representante de la UE. (2013). Comunicación Conjunta al Parlamento Europeo y al Consejo: El enfoque integral adoptado por la UE en relación con los conflictos y las crisis exteriores. Bruselas.

Consejo Europeo. (2003). Una Europa segura en un mundo mejor: Estrategia Europea de Seguridad. Bruselas.

Consejo Europeo. (2010, marzo, 25-26). Estrategia de Seguridad Interior de la Unión Europea: Hacia un Modelo Europeo de Seguridad. Bruselas.

De Carlos Izquierdo, C. (2016, septiembre, 30). La nueva Estrategia de Seguridad Europea 2016. Documento Marco n. ${ }^{\circ}$ 16. IEEE.

Haine, J. Y. (2008). The European Security Strategy Coping with Threats: Is Europe Secure? En S. Biscop \& J. J. Andersson (Eds.), The EU and the European Security Strategy: Forging a Global Europe. London: Routledge.

Salmon, T. (2004). 'United in its Diversity' (or Disunited in Adversary): That is the Question for the European Union and the European Security and Defence Policy. Perspectives on European Politics and Society, 5(3). DOI: https://doi.org/10.1080/15705850408438898

Servicio Europeo de Acción Exterior. (2016, junio). Shared Vision, Common Action: A Stronger Europe - A Global Strategy for the European Union's Foreign and Security Policy.

Tardy, T. (2009). The European Union, a Regional Security Actor with Global Aspirations. En T. Tardy (Ed.), European Security in a Global Context: Internal and External Dynamics. London: Routledge. DOI: https://doi.org/10.4324/9780203885352.pt1 